\title{
Adipogenesis: new insights into brown adipose tissue differentiation
}

\author{
Stefania Carobbio ${ }^{1,2}$, Barry Rosen ${ }^{1}$ and Antonio Vidal-Puig ${ }^{1,2}$ \\ ${ }^{1}$ Wellcome Trust Sanger Institute, Wellcome Trust Genome Campus, Hinxton, UK \\ ${ }^{2}$ Metabolic Research Laboratories, Addenbrooke's Treatment Centre, Institute of Metabolic Science, Addenbrooke's \\ Hospital, University of Cambridge, Cambridge, UK
}

Correspondence should be addressed

to A Vidal-Puig

Email

ajv22@medschl.cam.ac.uk

\begin{abstract}
Confirmation of the presence of functional brown adipose tissue (BAT) in humans has renewed interest in investigating the potential therapeutic use of this tissue. The finding that its activity positively correlates with decreased BMI, decreased fat content, and augmented energy expenditure suggests that increasing BAT mass/activity or browning of white adipose tissue (WAT) could be a strategy to prevent or treat obesity and its associated morbidities. The challenge now is to find a safe and efficient way to develop this idea.

Whereas BAT has being widely studied in murine models both in vivo and in vitro, there is an urgent need for human cellular models to investigate BAT physiology and functionality from a molecular point of view. In this review, we focus on the latest insights surrounding BAT development and activation in rodents and humans. Then, we discuss how the availability of murine models has been essential to identify BAT progenitors and trace their lineage. Finally, we address how this information can be exploited to develop human cellular models for BAT differentiation/activation. In this context, human embryonic stem and induced pluripotent stem cells-based cellular models represent a resource of great potential value, as they can provide a virtually inexhaustible supply of starting material for functional genetic studies, -omics based analysis and validation of therapeutic approaches. Moreover, these cells can be readily genetically engineered, opening the possibility of generating patient-specific cellular models, allowing the investigation of the influence of different genetic backgrounds on BAT differentiation in pathological or in physiological states.
\end{abstract}

\section{Key Words \\ - brown adipocytes \\ - brite adipocytes \\ - differentiation \\ - human stem cells}

Journal of Molecular

Endocrinology

(2013) 51, T75-T85

\section{Introduction}

The brown adipose tissue (BAT) is the main site of non-shivering thermogenesis in mammals and it responds to environmental stimuli such as cold and increased lipid-rich hypercaloric caloric intake, a process referred to as adaptive thermogenesis. This thermogenic role of BAT represents a specialised function mediated by a specific mitochondrial protein, uncoupling protein 1 (UCP1), which is located in the mitochondrial inner membrane, where it uncouples electron transport mitochondria respiration from ATP production, dissipating the proton motive force as heat - see review Cannon \& Nedergaard (2004).

Whereas the presence of functional active BAT in rodents has been known for many years, the realisation that BAT is also found in adult humans is much more recent. As in other mammals, BAT is an important organ controlling body temperature when humans are most vulnerable, at birth and early childhood (Lean 1989). However, several studies carried out in the last few years using biopsies following positron emission 
tomography coupled to computer tomography (PET-CT) imaging have confirmed the presence of thermogenically active BAT in adult humans (Cypess et al. 2009, van Marken Lichtenbelt et al. 2009, Zingaretti et al. 2009, Ouellet et al. 2011). These reports also showed some interesting correlations between BAT activation and decreased BMI, increased energy expenditure and decreased onset of diabetes.

The relevance of BAT activation to energy balance is well established in murine models. The main problem in adult humans is whether their relatively small amount of BAT may be sufficient to affect their energy balance. However, considering that in humans as little as $50 \mathrm{~g}$ of active BAT could account for a $20 \%$ increase of daily energy expenditure (Rothwell \& Stock 1983), these results support the concept that increasing BAT mass/activity or browning of white adipose tissue (WAT) could be a safe and efficient strategy to prevent or treat obesity (Bachman et al. 2002) and its associated complications. However, it is unclear what is the relevance of low temperatures to thermogenesis in humans as a recent study by Muzik and colleagues has shown that its contribution in subjects with relatively large BAT depots accounts only for $15-25 \mathrm{kcal} /$ day when they are exposed to mild cold temperatures $\left(15.5^{\circ} \mathrm{C}\right.$; Muzik et al. 2013). Hence, the promising therapeutic strategy is to discover treatments that increase and, more importantly, that activate BAT. The most common method of obtaining human brown adipocytes from human BAT is through biopsies, which tend to be both small and surgically difficult to obtain, giving access to a very limited numbers of cells. This limits the possibilities for investigating human BAT development, physiology, and activation from a molecular point of view: there is a need for good human BAT cellular models to enable application of these powerful approaches. The recent development of human embryonic stem (hES)- and induced pluripotent stem cells (IPS)-based cellular models for BAT and other cell types represent a promising opportunity to solve this problem.

On the basis of the potential therapeutic relevance of BAT, this review will focus on the latest knowledge surrounding the development and activation of BAT, both in rodents and in humans. Moreover, we will discuss the importance of the use of murine models to identify BAT precursors and trace their origins. Then, we will address how this information can be used to develop human cellular models based on hES and IPS cell technology for BAT differentiation/activation to be used in functional studies, -omics based analyses and validation of therapeutic approaches.

\section{A matter of adipocyte colours: brown, white, and beige/brite}

Until recently, the two main adipocyte cell types considered to form the mammalian adipose organ were identified as white and brown adipocytes. Despite both types of fat being able to accumulate substantial amount of lipids, they fulfil different functions; storage of fuel and mechanical protection in the case of the white and energy dissipation and heat production in the case of the brown. White adipose tissue is distributed in different depots along the body axis, i.e. visceral, subcutaneous, and perigonadal, whereas the BAT has a much more specific localisation. For instance, BAT in mice preferentially forms discrete depots in the interscapular region and around the aorta; however, brown adipocytes can also be identified as interspersed foci in skeletal muscle and within the white adipose tissue depots, i.e. subcutaneous and retriperitoneal (Cinti 2012). In adult humans, discrete BAT depots can be identified in the cervicalsupraclavicular area (Cypess et al. 2009), which are similar to the murine depots and are termed canonical BAT. In the last few years, several studies have reported the existence of a third type of adipose cell type, the brown in white ('brite') or 'beige' adipocyte. As in canonical BAT, the recruitment of the brite cells within the WAT tissue is induced by physiological thermogenic stimuli such as cold and/or high-caloric diet as well as by pharmacological treatments such as $\beta$-adrenergic agonists or thiazolidinedione (TZD) (Ohno et al. 2012, Wu et al. 2012). The development of brite/beige cells within WAT is called the 'browning' of WAT (Wu et al. 2012, Schulz et al. 2013) and can be observed by histological analysis showing increased numbers of adipocytes carrying characteristic multilocular lipid droplets dispersed within the WAT depots. These brite/beige cells present gene expression signatures similar to those of typical canonical brown adipocytes (i.e. Ucp1, Ppargc1a, and Cidea). These findings indicate that the brown adipocytes residing as interspersed foci within WAT depots are in fact brite cells. However, despite their similarities with canonical BAT, brite adipocytes also have specific gene expression patterns that are different from those of both white and canonical brown adipocytes. It is of note that, gene expression profiling performed by Walden et al. (2012) showed that the expression of Zic1 was quite specific to canonical BAT, whereas Hoxc9 was specific to brite and Tcf21 to white adipocytes. In an independent study, $\mathrm{Wu}$ et al. (2012) also identified Tmem26, Cd137, and Tbx1 as specific markers for brite cells. Interestingly, the same study suggested that brown and brite adipocytes exhibit selective responsiveness to specific differentiating factors such as

Published by Bioscientifica Ltd 
Bmp7 for the canonical BAT or irisin (a skeletal-musclederived myokine) for brite cells (Wu et al. 2012).

\section{Is human BAT brown or brite?}

Whether human BAT shares more genetic characteristics with mouse canonical brown or brite adipocytes has been an area of recent controversy. Wu and coworkers, based on gene expression profile of BAT biopsies from the supraclavicular region from two independent cohorts of human subjects, observed that human BAT from this anatomical location more resembled murine brite fat. These samples from the supraclavicular region had an enriched expression of Tmem26, Cd137, and Tbx1 genes that have been defined as specific markers for brite cells in mice (Wu et al. 2012). Similar results were obtained by Sharp et al. (2012) who investigated the genetic profile of multiple BAT depots located in the supraclavicular area, posterior mediastinum, retroperitoneal, intra-abdominal and mesenteric region of 13 human subjects.

However, in contrast, recent studies evaluating gene expression, differentiation capacity, and basal oxygen consumption analysis of biopsies isolated anatomical regions located deep in the neck fat (longus colli and carotid sheath area) from adult human volunteers showed that this depot shares many similarities with mouse canonical BAT (Cypess et al. 2013, Lidell et al. 2013). The bottom line is that humans seem to have both canonical and brite BAT at specific topographic locations. However, due to its position and anatomical diffuseness, the specific isolation of canonical BAT versus brite cells in humans by biopsies can be difficult, making gene expression experiments hard to interpret and control.

\section{Browning potential of WAT depots}

A relevant question is whether browning potential varies among different WAT depots? In support of an heterogenic response, $\mathrm{Wu}$ and colleagues were able to isolate 'brite' cells from inguinal and gonadal WAT of 129SVE mice and observed that in inguinal WAT expression of Ucp1 and other brown-adipocyte-enriched proteins, i.e. Cidea and Ppargc1a were mid-way between the levels observed in intrascapular canonical BAT and gonadal WAT. More importantly, despite basal expression of Ucp1 being low, adrenergic stimulation of brite cells in inguinal depot results in Ucp1 up-regulation to levels similar to those of canonical BAT. It is of relevance that the ability to activate brite cells in rodents also varies among different genetic backgrounds. Mice from a 129SVE obesity-resistant strain have detectable brite cells in the inguinal depot already at ambient temperature in the absence of cold exposure. Conversely, mice on a C56BL/6 obesity-prone genetic background require a strong adrenergic stimulus to induce brite cells in their WAT (Wu et al. 2012). Humans are also susceptible to browning of their WAT as shown by the anecdotal evidence that omental WAT biopsies obtained from patients affected by pheochromocytoma, a catecholamine-secreting tumour, are enriched in brite cells (Frontini et al. 2013).

\section{Tracking the developmental origins of canonical brown and brite cells}

Before embarking on manipulation of the amount/ activation of canonical brown fat or brite cells, it is essential to understand their origins in both embryo and adult - to address this question, the availability of both knockout and Cre recombinase-based lineage tracing mouse models has been instrumental.

\section{Origin of the canonical BAT}

BAT precursors in mouse embryo: $\mathrm{Myf5}^{+}$mesodermal lineage Classical BAT has been suggested to be derived from $\mathrm{Myf5}^{+}$progenitors from the paraxial mesoderm (Fig. 1) that share a common developmental origin with skeletal myoblasts. One of the key studies supporting this conclusion was performed by Seale and colleagues showing that the knockdown of the factor Prdm16 (PRD1F1-RIZ1 homologous domain containing 16) in brown adipocytes promoted myogenesis. This induction of skeletal muscle in cultures of brown fat pre-adipocytes suggested an initially surprising developmental closeness between these two mitochondria-rich cell lineages. To address this question more directly, lineage tracing experiments in mice were performed. Knock-in mice expressing Cre recombinase from the regulatory elements of the skeletal muscle-specific Myf5 gene were crossed with reporter mice that express yellow fluorescent protein (YFP) from the Rosa26 gene locus (R26R3-YFP) in a Cre-dependent manner. Immunohistological analysis of skeletal muscle, BAT and WAT from the interscapular region of 2- to 3-month-old Myf5-Cre:R26R3-YFP mice showed that YFP was highly expressed in skeletal muscle and BAT, but not in the WAT of these mice, confirming the common embryonic origin of the two tissues (Seale et al. 2008). In the mouse embryo, $\mathrm{Myf5}^{+}$precursors are located in the epaxial dermomyotome that derives from the paraxial mesoderm or presomitic mesoderm (PMS; Maroto et al. 2012). Lineage

Published by Bioscientifica Ltd 

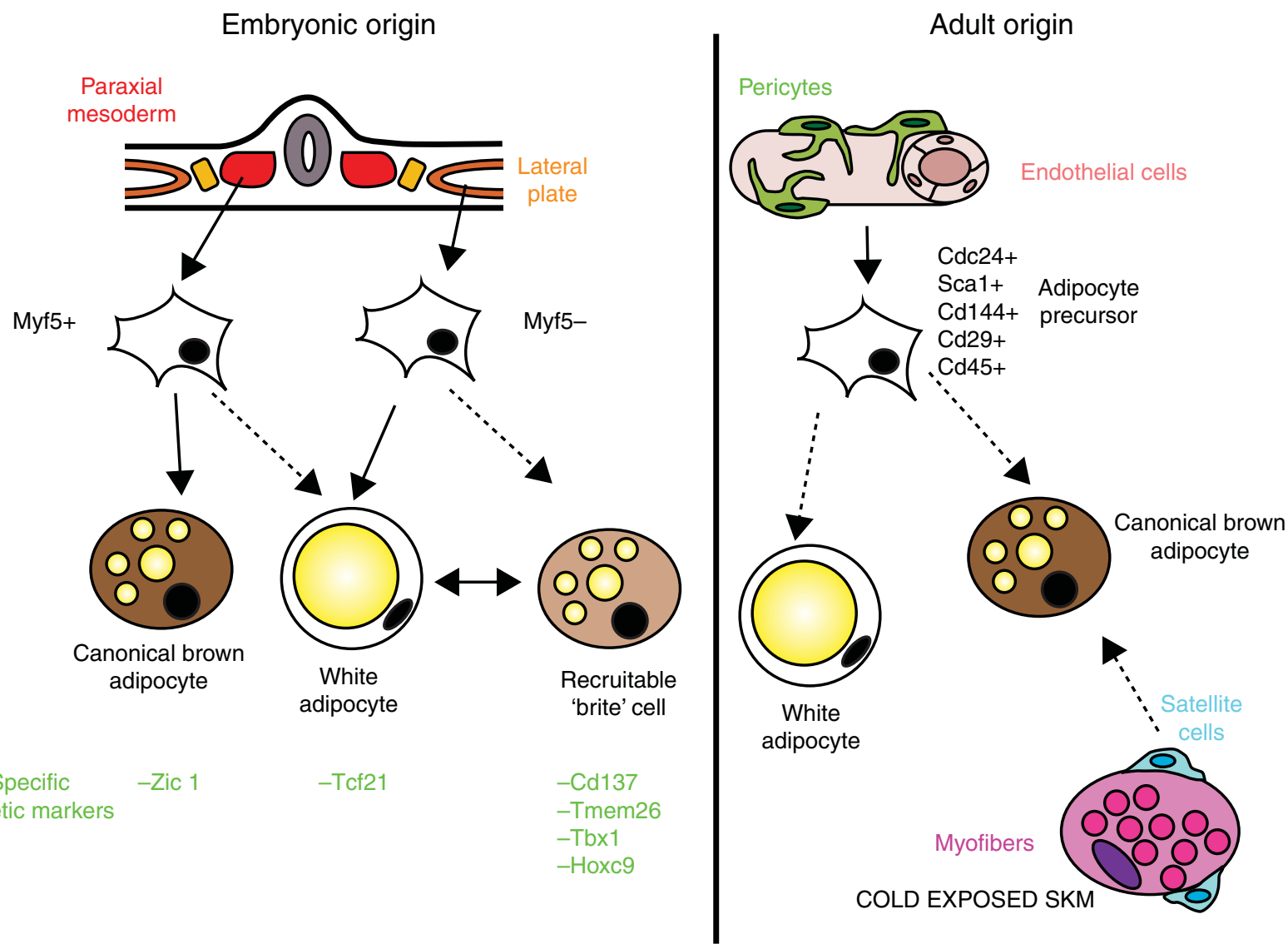

Figure 1

Overview of embryonic and adult origin of brown, brite, and white adipocytes indicating the specific cellular genetic markers.

tracing with Engrailed-1 (En1)-CreERT-inducible mouse crossed with a Rosa-floxed Stop-LacZ reporter also demonstrated that BAT originates from this embryonic region, En-1 being a marker for the dermomyotome. Epaxial muscle and dermis have been demonstrated to arise from this same En1-positive embryonic region (Atit et al. 2006).

Further evidence for the 'myogenic' developmental origin of BAT came from knockout mouse studies. Doubleknockout mice for Meox1 and Meox2, transcription factors expressed in the somites, which play an essential role in somite patterning and subsequent myogenic development, showed impaired epaxial muscle formation associated with the absence of the overlying BAT, suggesting origins from a common precursor pool (Mankoo et al. 2003).

Identification of Myf5-positive precursors for white adipose tissue In a recent study, SanchezGurmaches and colleagues investigated the impact of conditional phosphatase and tensin homologue (PTEN) depletion on the growth of mesoderm-derived tissues, using the same Myf5-Cre knock-in Cre allele used previously to trace brown adipocyte origin. The Myf5Cre:PTEN conditional knockout showed severely overgrown intrascapular, subscapular, and cervical BATs, consistent with brown adipocytes originating from Myf5-positive lineage. However, in contradiction to the model suggesting that all white adipocytes derive from a Myf5-negative precursor, the Myf5-PTEN conditional knockout also had hypertrophic anterior, subcutaneous, and retroperitoneal WAT, coupled with a complete absence of perigonadal, mesenteric, and posterior subcutaneous WAT (Sanchez-Gurmaches et al. 2012). The total redistribution of body fat in this mouse suggests the heterogeneity of the white adipose tissue depots and the possibility that the Myf5 lineage may contribute to white adipocyte development in specific depots (Fig. 1). The contribution of the Myf5 lineage to adipose tissue was reanalysed by this group, using the Myf5-Cre knock-in allele, which was again combined with the R26R-YFP reporter and also separately with the R26R-LacZ reporter, and lineage tracing was performed on a broad selection of WAT depots. The result was that as

Published by Bioscientifica Ltd 
expected, Myf5 was found to drive the expression of YFP and LacZ not only in classical BAT depots (intrascapular, supraclavicular, and cervical) but also in retroperitoneal and anterior subcutaneous WATs as well as, and to a lesser extent in inguinal and perigonadal WAT (SanchezGurmaches et al. 2012). In an independent study, Shan et al. (2013) confirmed the existence of Myf5-positive cells in WAT. This discrepancy may simply reflect that when the first BAT lineage tracing with Myf5-Cre knock-in was performed, only a few adipose tissue depots were analysed, resulting in an underestimation of the contribution of the Myf5 lineage to the whole fat organ development.

\section{BAT precursors in adult mice: endothelial and other origins}

Multiple studies have identified several early progenitors from adult mouse tissues that seem to be able to differentiate both into BAT and WAT. Recently, work by Tran and colleagues reported that murine endothelial cells (ECs) of classic white and brown fat depots share ultrastructural characteristics with pericytes, a stem celllike population that can potentially differentiate into preadipocytes. Lineage tracing experiment using VE-cadherin promoter revealed localisation of reporter genes in not only pre-adipocytes and adipocytes of WAT and BAT depots but also in ECs (Tran et al. 2012). Taken together, these results support an endothelial origin of murine and human adipocytes (Fig. 1), suggesting a model for how adipogenesis and angiogenesis are regulated to coordinate adipose tissue expansion. Using lineage tracing, Zfp423 has recently been identified as a factor playing a key role in the early commitment of both brown and white fat. Zpf423 is easily detected in both BAT and WAT preadipocyte depots in the adipose vasculature, coinciding with a subset of pericytes (Gupta et al. 2012), suggesting once again a possible endothelial origin of fat. In an independent study, WAT precursors in adipose vasculature were also identified by Tang et al. (2008). However, these results have been recently contradicted by the results of experiments by Berry \& Rodeheffer (2013), which showed that a variety of endothelial/haematopoietic Cre drivers did not result in labelling of the adipocyte lineages. Previous evidence for a perivascular adipocyte precursor may have been complicated by the difficulties of localising markers to either type of sparse adipocyte cytoplasm compared with juxtaposed precursor cells, highlighting the practical caveats of the in vivo mouse lineage tracing system. Recent evidence also indicates that brown adipocytes may arise in adult skeletal muscle from satellite cells during cold exposure via a micro-RNA-mediated pathway (Fig. 1; Yin et al. 2013).

\section{The origins of the recruitable BAT (brite)}

Brite adipocytes seem to share a common origin with white adipocytes through either transdifferentiation from WAT to BAT following adrenergic stimulation or alternatively by development from bipotential common early progenitors. In support of the transdifferentiation hypothesis, Himms-Hagen and colleagues showed that rats treated for 1 week with CL-316243, a $\beta 3$-adrenoreceptor agonist, develop multilocular mitochondria-rich adipocytes in retroperitoneal WAT depot arising mostly from direct conversion of a subpopulation of pre-existing unilocular white adipocytes. Those newly formed brownlike adipocytes have morphological and mitochondrial protein patterns that can be considered intermediate between those of classical WAT and BAT (Himms-Hagen et al. 2000). Lineage tracing studies in mice with an inducible Ucp1 Cre recombinase have confirmed not only that chronic cold induces formation of brite adipocytes from the inguinal WAT depot but also that it can be reversed within 5 weeks of warm adaptation, suggesting that the transdifferentiation between white and brite adipocytes is bidirectional depending on environmental exposure (Rosenwald et al. 2013).

Supporting the hypothesis of a common bipotential cell precursor, Lee et al. reported the isolation of PDGFR $\alpha^{+}$ $\mathrm{Cd}^{+}{ }^{+} \mathrm{Sca} 1^{+}$early precursors from murine WAT that were able to differentiate into both BAT and WAT in vitro depending on the inductive cues, such as high-fat diet and ADR stimulation. It is of note that these precursors are able to self-renew (Lee et al. 2012b). Moreover, Li and colleagues have reported that, in epididymal fat following CL316243 treatment, multilocular cells are generated by stimulation of cell proliferation and increased catabolic capacity (Li et al. 2005).

In conclusion, whether the appearance of brite cells within white adipose tissue in response to thermogenic stimuli is due to transdifferentiation of white adipocytes to brite cells and/or from differentiation from a common progenitor residing within the WAT depots (or both) still requires further investigation.

\section{Cellular models of human brown/brite differentiation}

Recent attention to human BAT and species differences between rodents and humans regarding their thermogenic

Published by Bioscientifica Ltd 
capacity, pharmacological responsiveness and marker gene expression (Langin 2010, Pisani et al. 2011) highlights the need for more extensive studies elucidating the biology of human BAT. Human BAT is scarce and difficult to obtain and there are few well-characterised human brown adipocyte cellular models. Below we discuss both current cellular models and the new possibilities emerging from hES/IPS cell-based models for investigating human BAT physiology and differentiation.

\section{Immortalised cell line isolated from human BAT}

So far, the only cell line derived from human BAT described in the literature is the PAZ6 cell line, which was obtained from the stromal vascular fraction of infant BAT (Zilberfarb et al. 1997). This cell line recapitulates many features of mature brown adipocytes (reviewed in Beranger et al. (2013)). However, the protocol used to immortalise this cell line required infection with SV40 large $\mathrm{T}$ antigen, which binds retinoblastoma protein (pRB) and relieves the pRB control of the cell cycle (Ali \& DeCaprio 2001). pRB functions as a molecular switch driving cells towards the white instead of towards the brown phenotype; thus, it is not surprising that its inactivation directs these cells to acquire brown characteristics both in vivo and in vitro (Hansen et al. 2004). It cannot be excluded that the immortalisation protocol could interfere with pRB functionality in these cells. Furthermore, the differentiation of PAZ6 into brown adipocytes requires the permanent addition of high concentration of TZD and it is known that chronic treatment with this compound facilitates the development of 'brite' cells (Petrovic et al. 2010). So PAZ6 cells could represent a mixed brown/brite phenotype, making them unsuitable for dissecting the molecular mechanisms characterising these two cell types.

\section{Primary cultures derived human adult BAT}

Primary cultures from adult tissue have been widely used to investigate brown adipocyte differentiation and can be considered to be the closest model to in vivo BAT (Cannon \& Nedergaard 2004, Skurk \& Hauner 2012). However, there are evident limitations concerning this approach, most of all the small number of cells that can be obtained from human BAT biopsies. Despite this problem, there are reports describing the use of human primary cells to study brown adipocyte differentiation (Lee et al. 2011).

\section{Human adipose-derived stem cells}

Several studies have been published investigating the potential of human multipotent adipose-derived stem cells (hMADS) as precursors for differentiation into brown or brite adipocytes. For instance, Elabd and colleagues investigated the capacity of hMADS obtained from paediatric subcutaneous adipose tissue biopsies to become brown adipocytes. Whereas short-term exposure of hMADS to the peroxisome proliferator-activated receptor $\gamma(\operatorname{PPAR} \gamma)$ agonist rosiglitazone induced their differentiation to white adipocytes, chronic treatment with this compound stimulated the development of brown-adipose-like cells (Elabd et al. 2009). Moreover, treatment of hMADS with T3 hormone, a well-known activator thermogenesis, or with natriuretic peptides, promotes the expression of UCP1, PPARGC1A, and other factors known to promote mitochondriogenesis and mitochondrial respiration (Bordicchia et al. 2012, Lee et al. 2012a). Globally considered, this suggests that hMADS could possibly represent a suitable model to study the conversion of white mature adipocyte to brown-adipose-like, although cell numbers obtained are again limited.

\section{Human adult multipotent mesenchymal precursors}

Another potential biological source to investigate brown adipocyte differentiation is mesenchymal precursors cells (MPCs) also called 'mesenchymal stem cells' that are multipotent stromal cells present in various adult tissues, e.g. bone marrow (Huang et al. 2011) and foetal muscle (Crisan et al. 2008), that can be isolated by selection using surface markers (i.e. $\mathrm{CD}_{3}{ }^{+}, \mathrm{CD} 105^{+}, \mathrm{CD} 19^{-}$, and $\mathrm{CD} 45^{-}$) or cell adhesion. MPCs are able to differentiate into different cell types such as adipocytes, chondrocytes, osteoblasts, and sometimes myoblasts.

\section{The future: hES and IPS cell-based differentiation models}

The previously described human adult and foetal derived BAT cell culture systems have limited proliferative capacity, heterogeneous, and progressively impaired differentiation and cannot be used to reliably produce large numbers of pure brown adipocytes. To surmount these issues, several groups have utilized in vitro differentiation of hES and IPS cells to generate models of human brown and white adipogenesis. These pluripotent stem cells present distinct advantages for in vitro modelling of adipocyte function: i) they can self-renew and thus represent a virtually

Published by Bioscientifica Ltd 
inexhaustible supply of starting material; ii) they are normal diploid cells; iii) it is possible to derive patient-specific disease models of known genotypes with IPS technology; iv) pluripotent cells can be simultaneously differentiated into several metabolically relevant lineages (e.g. brown and white adipocytes, hepatocytes, skeletal muscle, and neurons); and v) they can be routinely genetically engineered to introduce various types of mutations or make reporter cell lines. These studies have benefited from a growing practical knowledge of both in vitro differentiation and genome engineering in pluripotent human stem cells.

\section{Generation of multipotent MPCs from human stem cells}

Protocols developed for the differentiation of hIPS/ES cells into fully differentiated and functional cells from all three embryonic germ layers can involve a combination of manipulating key signalling pathways with pharmacological agents (both small molecules and growth factors), mimicking normal developmental processes, and enforced expression of key lineage-specific transcription factors to drive cells to specific cell fates. For the adipocyte lineages, a key intermediate cell type is the MPC, which is characterised by expression of a suite of surface markers (CD73, CD105, etc.) shared with adult in vivo-derived MPCs (described earlier) and the ability to differentiate to adipocytes, chondrocytes and osteocytes (and sometimes skeletal muscle cells) under appropriate conditions. MPCs proliferate rapidly under conditions where other ES cell-derived differentiated cells do not and can be expanded through many $(10+)$ passages under simple growth conditions (fetal calf serum plus bovine fibroblast growth factor) making them an amplifiable source of adipocyte precursors. A number of protocols have been published for the derivation of homogenous populations of MPC's from both hES and IPS cells using either supporting cell (OP9) monolayers (Barberi et al. 2005) or forced cell aggregates called embryoid bodies followed by fluorescence-activated cell sorting for MPC-specific markers as a starting point (Ahfeldt et al. 2012, de Peppo et al. 2013). However, MPCs display only partial and heterogeneous differentiation to adipocytes and do not themselves represent a practical model system for obtaining large number of homogeneous adipocytes.

More recently, there have been several groups developing step-by-step protocols mimicking the different states of embryonic mesoderm differentiation using the factors/morphogens and signalling pathways involved in embryonic paraxial mesoderm development, i.e. FGF, Bmps, Wnt, and Shh signalling (Francetic \& Li 2011, Cheung et al. 2012, Sakurai et al. 2012).

\section{Brown adipocytes differentiated from hES and IPS cells}

Two prominent recent studies have employed hES and/or IPS cells to generate functional models of BAT differentiation. Two different approaches have been developed to obtain mature brown adipocytes from these cells: i) expression of adipogenic transcription factors and ii) signalling pathway manipulation with growth factors.

Ahfeldt et al. (2012) developed a transgeneexpression-based approach to obtain highly differentiated and functional brown and white adipocytes from both hES and IPS cells. Their system involves first differentiating human stem cells to MPCs by embryoid body formation and enrichment of this population by serial passage in serum and FGF followed by expression of adipogenic transcription factors via inducible (Tet system) lentiviral constructs to induce adipocyte terminal differentiation. Expression of PPARg2 alone results in very efficient conversion $(>85 \%)$ to white adipocytes while combining PPARg2 with C/EBP $\beta$ and PRDM16 (non-essential) results in homogeneous and efficient brown adipocyte differentiation. The brown adipocytes programmed in this way exhibited a bona fide BAT gene expression profile and mature brown adipocytes displayed functional properties such as increased lipolysis in response to $\beta$-adrenergic stimulation and oxygen consumption levels. Stable, faithful maintenance of both the brown and white adipocyte phenotypes could be achieved even after inducible ectopic transcription factor expression was switched off. Furthermore, these programmed brown adipocytes were functional in vivo when transplanted into genetically immunocompromised mice, displaying histological characteristics of primary BAT morphologically and glucose uptake in a PET-CT scan (Ahfeldt et al. 2012).

Nishio et al. (2012) developed an alternative approach to reprogram hES and IPS cells into functional brown adipocytes both in vitro and in vivo with an efficiency of $>90 \%$ without utilizing exogenous gene transfer. The protocol was initially discovered by observations that conditions which were established to differentiate cells of the haematopoietic lineage resulted in the production of significant numbers of brown adipocytes (Thorns et al. 2008, Krings et al. 2012). They employed a haemopoietic cocktail (HC) composed of KIT ligand (KITGL), fms-related tyrosine kinase 3 ligand (FLT3LG), interleukin 6 (IL6), and VEGF along with the previously reported BAT inducer BMP7 (Tseng et al. 2008). When applied to hES and IPS cells, these factors drove efficient differentiation into brown adipocytes in a protocol that includes

Published by Bioscientifica Ltd 
aggregation in semisolid medium in the presence of mesoderm-inducing factors like BMP4 in HC followed by adherent culture in HC. The differentiated brown adipocyte cells exclusively contained multilocular lipid droplets and quantitative RT-PCR confirmed the induction of BAT-specific genes such as UCP1 and PRDM16. This study also evaluated functional maturation by treating them with a $\beta$-adrenergic agonist, isoproterenol and looking at UCP1 and PRDM16 expression, which were further increased compared with basal levels. Moreover, these cells showed increased respiration and oxygen consumption rates after induction with a specific $\beta 3$-adrenergic agonist CL316243. Transplantation of the reprogrammed brown adipocytes in mice improved fasting TG, blood glucose levels, and glucose tolerance versus mice that received transplants of immature IPS cells, proving the in vitro stem-cell-derived BAT was capable of in vivo function. Furthermore, transplantation of in vitro-matured BAT cells ameliorated the detrimental metabolic effects of WAT transplantation, proving that these in vitro-matured cells can have therapeutic effects in vivo.

Although these studies are groundbreaking for opening a new approach for studying brown adipocyte formation and function, the reported differentiation protocols have been applied to only a limited number of hES and hIPS lines so far and their general applicability to human pluripotent cell lines has yet to be determined.

\section{Genome engineering of hES/IPS cells}

The ability to selectively introduce or correct mutations in the genome of stem-cell-derived human brown adipocytes represents a powerful technology for interrogating and understanding gene function. Genetically engineering human stem cells has become more approachable in the past few years with the introduction of new powerful 'designer nuclease' technologies such as transcription activator-like effectors nucleases (TALENS; Cermak et al. 2011) and RNA guided endonucleases (RGENs) such as the Cas9/CRISPR system (Ding et al. 2013b, Gaj et al. 2013). These powerful tools can be used both to inactivate one or even both alleles of a gene in stem cells in a single step by non-homologous end joining or to introduce subtle mutations and/or insertions (such as reporter genes) via homologous recombination (HR). One of the most powerful applications of these designer nucleases is the ability to create matched, isogenic cell lines for examining the effects of mutations on cellular phenotypes by either creating disease mutations in normal lines or correcting them in lines derived from affected patients to create an appropriate control line (Ding et al. 2013b).

\section{Cellular phenotypes in adipocytes differentiated from engineered stem cells}

Analysis of cellular phenotypes has the potential to be used to dissect genetic effects into their causal components directly in metabolically relevant human cell types differentiated from pluripotent human stem cells. The power and versatility of this approach has been persuasively demonstrated by Ding et al. (2013a) in a report in which they describe how they efficiently generated mutant alleles of 15 genes in human pluripotent stem cells with TALENS and differentiated the engineered cells containing a variety of null and subtle mutant alleles into various metabolically relevant cell types including white adipocytes, hepatocyte-like cells and neurons. Null mutations in the sortilin (SORT1) gene, originally identified in genomewide association studies of determinants of LDL cholester$\mathrm{ol} /$ coronary disease, were demonstrated to have significant effects on insulin-responsive glucose transport in white adipocytes and ApoB secretion in hepatocytes, as well as effects on BDNF-mediated cell death in neurons. An allelic series for the $A K T 2$ gene that included a null mutation and dominant patient-derived point mutation (constructed by oligonucleotide-mediated HR with TALENs) was introduced into hES cells, and the two types of mutations revealed opposing phenotypic effects on glucose uptake, triglyceride content and adipokine secretion in white adipocytes, as well as effects on glucose production in hepatocytes. Similar studies in ES/IPS-derived brown adipocytes should become a powerful method for both interrogating the function of various metabolically relevant genes and testing the effects of putative human mutations on BAT function in a highly sensitive and wellcontrolled cellular system.

\section{Future opportunities}

hES and IPS cells represent very powerful and accessible tools for studying human BAT differentiation and activation. They should enable the generation of virtually unlimited amounts of cellular material that can be used as platforms for different types of screening, i.e. for new potential drugs activating BAT, for genomic studies and other -omics analysis to identify new candidate genes or metabolites important for BAT development/activity in both healthy and diseased states. The purification/isolation of highly pure brown adipocytes should be facilitated by the ability to

Published by Bioscientifica Ltd 


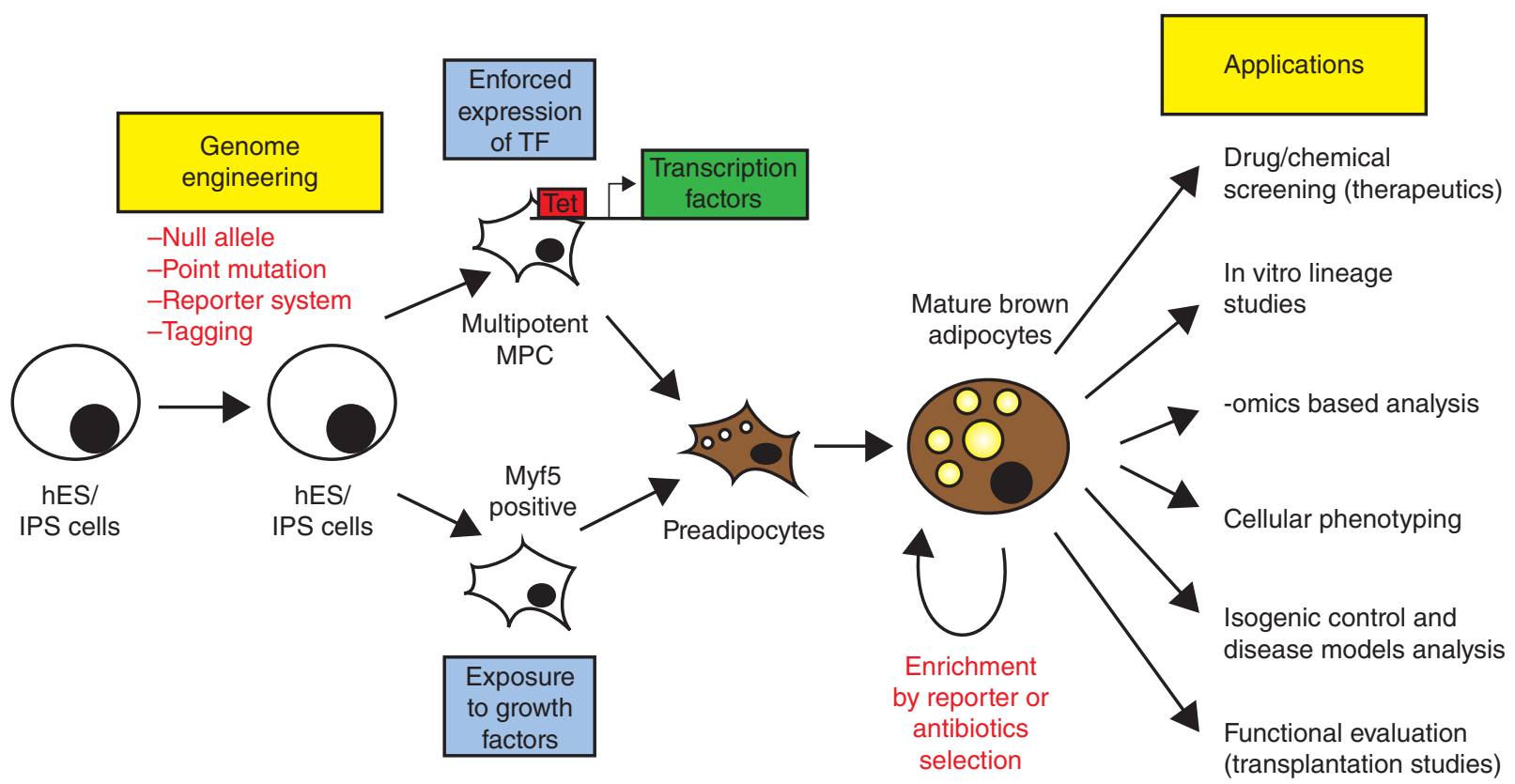

Figure 2

Summary of the protocols for differentiation of $\mathrm{hES} / \mathrm{IPS}$ into brown adipose tissue and possible applications of the $\mathrm{hES} / \mathrm{IPS}$ cellular systems. TF, transcription factors; hES, human embryonic stem cells; hIPS, human induced pluripotent stem cells; MPC, mesenchymal precursor cells.

engineer stem cell lines in which key markers of the BAT lineage are marked with fluorescent, antibiotic or neutral cell-surface reporters. The combination of genetic lineage marking using site-specific recombinases like Cre and highly defined human stem cell differentiation systems that mimic the progression of paraxial mesoderm formation during embryonic development (Francetic \& Li 2011, Cheung et al. 2012, Sakurai et al. 2012) may help to resolve some of the outstanding controversial questions regarding the origin of both human classical BAT and brite cells and test the importance of various factors in directing BAT/brite cell fate. Engineered reporter lines could also be employed in largescale chemical screening for compounds that influence BAT formation/activity and are potential therapeutic drugs for metabolic disease. Furthermore, the possibility of establishing hIPS cell lines from different human subjects guarantees the possibility of studying the influence of different genetic backgrounds on BAT differentiation/activity. Finally, animal transplantation-based systems can also be developed to test stem-cell-derived human BAT cells in vivo to evaluate the function of the cells in a physiological environment (Nishio et al. 2012; Fig. 2).

\section{Conclusion}

In summary, activation of BAT in humans offers a unique opportunity to treat obesity and its metabolic consequences. However, the success of this strategy requires more knowledge about the development and activation of human BAT and good cellular models. To elucidate this, the combination of insights into BAT development from rodent models combined with the development of hES and IPS in vitro differentiation models that can be readily engineered and functionally interrogated offer a unique opportunity to overcome the limitations imposed by lack of suitable biological material opening unprecedented opportunities to tackle the problem of obesity and the metabolic syndrome.

Declaration of interest

The authors declare that there is no conflict of interest that could be perceived as prejudicing the impartiality of the review reported.

\section{Funding}

This work was supported by FP7 BetaBAT, BBSRC, and MRC programme grant and by the Wellcome Trust.

\section{References}

Ahfeldt T, Schinzel RT, Lee YK, Hendrickson D, Kaplan A, Lum DH, Camahort R, Xia F, Shay J, Rhee EP et al. 2012 Programming human pluripotent stem cells into white and brown adipocytes. Nature Cell Biology 14 209-219. (doi:10.1038/ncb2411)

Published by Bioscientifica Ltd 
Ali SH \& DeCaprio JA 2001 Cellular transformation by SV40 large T antigen: interaction with host proteins. Seminars in Cancer Biology 11 15-23. (doi:10.1006/scbi.2000.0342)

Atit R, Sgaier SK, Mohamed OA, Taketo MM, Dufort D, Joyner AL, Niswander L \& Conlon RA $2006 \beta$-Catenin activation is necessary and sufficient to specify the dorsal dermal fate in the mouse. Developmental Biology 296 164-176. (doi:10.1016/j.ydbio.2006.04.449)

Bachman ES, Dhillon H, Zhang CY, Cinti S, Bianco AC, Kobilka BK \& Lowell BB $2002 \beta A R$ signaling required for diet-induced thermogenesis and obesity resistance. Science 297 843-845. (doi:10.1126/science.1073160)

Barberi T, Willis LM, Socci ND \& Studer L 2005 Derivation of multipotent mesenchymal precursors from human embryonic stem cells. PLoS Medicine 2 e161. (doi:10.1371/journal.pmed.0020161)

Beranger GE, Karbiener M, Barquissau V, Pisani DF, Scheideler M, Langin D \& Amri EZ 2013 In vitro brown and "brite"/"beige" adipogenesis: human cellular models and molecular aspects. Biochimica et Biophysica Acta 1831 905-914. (doi:10.1016/j.bbalip.2012.11.001)

Berry R \& Rodeheffer MS 2013 Characterization of the adipocyte cellular lineage in vivo. Nature Cell Biology 15 302-308. (doi:10.1038/ncb2696)

Bordicchia M, Liu D, Amri EZ, Ailhaud G, Dessi-Fulgheri P, Zhang C, Takahashi N, Sarzani R \& Collins S 2012 Cardiac natriuretic peptides act via $\mathrm{p} 38 \mathrm{MAPK}$ to induce the brown fat thermogenic program in mouse and human adipocytes. Journal of Clinical Investigation 122 1022-1036. (doi:10.1172/JCI59701)

Cannon B \& Nedergaard J 2004 Brown adipose tissue: function and physiological significance. Physiological Reviews 84 277-359. (doi:10.1152/physrev.00015.2003)

Cermak T, Doyle EL, Christian M, Wang L, Zhang Y, Schmidt C, Baller JA, Somia NV, Bogdanove AJ \& Voytas DF 2011 Efficient design and assembly of custom TALEN and other TAL effector-based constructs for DNA targeting. Nucleic Acids Research 39 e82. (doi:10.1093/nar/gkr218)

Cheung C, Bernardo AS, Trotter MW, Pedersen RA \& Sinha S 2012 Generation of human vascular smooth muscle subtypes provides insight into embryological origin-dependent disease susceptibility. Nature Biotechnology 30 165-173. (doi:10.1038/nbt.2107)

Cinti S 2012 The adipose organ at a glance. Disease Models \& Mechanisms 5 588-594. (doi:10.1242/dmm.009662)

Crisan M, Casteilla L, Lehr L, Carmona M, Paoloni-Giacobino A, Yap S, Sun B, Leger B, Logar A, Penicaud L et al. 2008 A reservoir of brown adipocyte progenitors in human skeletal muscle. Stem Cells $\mathbf{2 6}$ 2425-2433. (doi:10.1634/stemcells.2008-0325)

Cypess AM, Lehman S, Williams G, Tal I, Rodman D, Goldfine AB, Kuo FC, Palmer EL, Tseng YH, Doria A et al. 2009 Identification and importance of brown adipose tissue in adult humans. New England Journal of Medicine 360 1509-1517. (doi:10.1056/NEJMoa0810780)

Cypess AM, White AP, Vernochet C, Schulz TJ, Xue R, Sass CA, Huang TL, Roberts-Toler C, Weiner LS, Sze C et al. 2013 Anatomical localization, gene expression profiling and functional characterization of adult human neck brown fat. Nature Medicine 19 635-639. (doi:10.1038/ $\mathrm{nm} .3112)$

Ding Q, Lee YK, Schaefer EA, Peters DT, Veres A, Kim K, Kuperwasser N, Motola DL, Meissner TB, Hendriks WT et al. 2013a A TALEN genomeediting system for generating human stem cell-based disease models. Cell Stem Cell 12 238-251. (doi:10.1016/j.stem.2012.11.011)

Ding Q, Regan SN, Xia Y, Oostrom LA, Cowan CA \& Musunuru K 2013b Enhanced efficiency of human pluripotent stem cell genome editing through replacing TALENs with CRISPRs. Cell Stem Cell 12 393-394. (doi:10.1016/j.stem.2013.03.006)

Elabd C, Chiellini C, Carmona M, Galitzky J, Cochet O, Petersen R, Penicaud L, Kristiansen K, Bouloumie A, Casteilla L et al. 2009 Human multipotent adipose-derived stem cells differentiate into functional brown adipocytes. Stem Cells 27 2753-2760. (doi:10.1002/stem.200)

Francetic T \& Li Q 2011 Skeletal myogenesis and Myf5 activation. Transcription 2 109-114. (doi:10.4161/trns.2.3.15829)

Frontini A, Vitali A, Perugini J, Murano I, Romiti C, Ricquier D, Guerrieri M \& Cinti S 2013 White-to-brown transdifferentiation of omental adipocytes in patients affected by pheochromocytoma. Biochimica et Biophysica Acta 1831 950-959. (doi:10.1016/j.bbalip.2013.02.005)

Gaj T, Gersbach CA \& Barbas CF III 2013 ZFN, TALEN, and CRISPR/ Cas-based methods for genome engineering. Trends in Biotechnology 31 397-405. (doi:10.1016/j.tibtech.2013.04.004)

Gupta RK, Mepani RJ, Kleiner S, Lo JC, Khandekar MJ, Cohen P, Frontini A, Bhowmick DC, Ye L, Cinti S et al. 2012 Zfp423 expression identifies committed preadipocytes and localizes to adipose endothelial and perivascular cells. Cell Metabolism 15 230-239. (doi:10.1016/j.cmet. 2012.01.010)

Hansen JB, Jorgensen C, Petersen RK, Hallenborg P, De Matteis R, Boye HA, Petrovic N, Enerback S, Nedergaard J, Cinti S et al. 2004 Retinoblastoma protein functions as a molecular switch determining white versus brown adipocyte differentiation. PNAS 101 4112-4117. (doi:10.1073/ pnas.0301964101)

Himms-Hagen J, Melnyk A, Zingaretti MC, Ceresi E, Barbatelli G \& Cinti S 2000 Multilocular fat cells in WAT of CL-316243-treated rats derive directly from white adipocytes. American Journal of Physiology. Cell Physiology 279 C670-C681.

Huang PI, Chen YC, Chen LH, Juan CC, Ku HH, Wang ST, Chiou SH, Chiou GY, Chi CW, Hsu CC et al. 2011 PGC-1 $\alpha$ mediates differentiation of mesenchymal stem cells to brown adipose cells. Journal of Atherosclerosis and Thrombosis 18 966-980. (doi:10.5551/jat.7401)

Krings A, Rahman S, Huang S, Lu Y, Czernik PJ \& Lecka-Czernik B 2012 Bone marrow fat has brown adipose tissue characteristics, which are attenuated with aging and diabetes. Bone 50 546-552. (doi:10.1016/ j.bone.2011.06.016)

Langin D 2010 Recruitment of brown fat and conversion of white into brown adipocytes: strategies to fight the metabolic complications of obesity? Biochimica et Biophysica Acta 1801 372-376. (doi:10.1016/ j.bbalip.2009.09.008)

Lean ME 1989 Brown adipose tissue in humans. Proceedings of the Nutrition Society 48 243-256. (doi:10.1079/PNS19890036)

Lee P, Swarbrick MM, Zhao JT \& Ho KK 2011 Inducible brown adipogenesis of supraclavicular fat in adult humans. Endocrinology 152 3597-3602. (doi:10.1210/en.2011-1349)

Lee JY, Takahashi N, Yasubuchi M, Kim YI, Hashizaki H, Kim MJ, Sakamoto T, Goto T \& Kawada T 2012a Triiodothyronine induces UCP-1 expression and mitochondrial biogenesis in human adipocytes. American Journal of Physiology. Cell Physiology 302 C463-C472. (doi:10.1152/ajpcell.00010.2011)

Lee YH, Petkova AP, Mottillo EP \& Granneman JG 2012b In vivo identification of bipotential adipocyte progenitors recruited by $\beta 3$-adrenoceptor activation and high-fat feeding. Cell Metabolism 15 480-491. (doi:10.1016/j.cmet.2012.03.009)

Li P, Zhu Z, Lu Y \& Granneman JG 2005 Metabolic and cellular plasticity in white adipose tissue II: role of peroxisome proliferator-activated receptor- $\alpha$. American Journal of Physiology. Endocrinology and Metabolism 289 E617-E626. (doi:10.1152/ajpendo.00010.2005)

Lidell ME, Betz MJ, Leinhard OD, Heglind M, Elander L, Slawik M, Mussack T, Nilsson D, Romu T, Nuutila P et al. 2013 Evidence for two types of brown adipose tissue in humans. Nature Medicine 19 631-634. (doi:10.1038/nm.3017)

van Marken Lichtenbelt WD, Vanhommerig JW, Smulders NM, Drossaerts JM, Kemerink GJ, Bouvy ND, Schrauwen P \& Teule GJ 2009 Cold-activated brown adipose tissue in healthy men. New England Journal of Medicine 360 1500-1508. (doi:10.1056/NEJMoa0808718)

Mankoo BS, Skuntz S, Harrigan I, Grigorieva E, Candia A, Wright CV, Arnheiter H \& Pachnis V 2003 The concerted action of Meox homeobox genes is required upstream of genetic pathways essential for the formation, patterning and differentiation of somites. Development 130 4655-4664. (doi:10.1242/dev.00687)

Maroto M, Bone RA \& Dale JK 2012 Somitogenesis. Development 139 2453-2456. (doi:10.1242/dev.069310)

Muzik O, Mangner TJ, Leonard WR, Kumar A, Janisse J \& Granneman JG 201315 O PET measurement of blood flow and oxygen consumption 
in cold-activated human brown fat. Journal of Nuclear Medicine $\mathbf{5 4}$ 523-531. (doi:10.2967/jnumed.112.111336)

Nishio M, Yoneshiro T, Nakahara M, Suzuki S, Saeki K, Hasegawa M, Kawai Y, Akutsu H, Umezawa A, Yasuda K et al. 2012 Production of functional classical brown adipocytes from human pluripotent stem cells using specific hemopoietin cocktail without gene transfer. Cell Metabolism 16 394-406. (doi:10.1016/j.cmet.2012.08.001)

Ohno H, Shinoda K, Spiegelman BM \& Kajimura S 2012 PPAR $\gamma$ agonists induce a white-to-brown fat conversion through stabilization of PRDM16 protein. Cell Metabolism 15 395-404. (doi:10.1016/j.cmet. 2012.01.019)

Ouellet V, Routhier-Labadie A, Bellemare W, Lakhal-Chaieb L, Turcotte E, Carpentier AC \& Richard D 2011 Outdoor temperature, age, sex, body mass index, and diabetic status determine the prevalence, mass, and glucose-uptake activity of 18F-FDG-detected BAT in humans. Journal of Clinical Endocrinology and Metabolism 96 192-199. (doi:10.1210/ jc.2010-0989)

de Peppo GM, Marcos-Campos I, Kahler DJ, Alsalman D, Shang L, Vunjak-Novakovic G \& Marolt D 2013 Engineering bone tissue substitutes from human induced pluripotent stem cells. PNAS 110 8680-8685. (doi:10.1073/pnas.1301190110)

Petrovic N, Walden TB, Shabalina IG, Timmons JA, Cannon B \& Nedergaard J 2010 Chronic peroxisome proliferator-activated receptor $\gamma(\operatorname{PPAR} \gamma)$ activation of epididymally derived white adipocyte cultures reveals a population of thermogenically competent, UCP1-containing adipocytes molecularly distinct from classic brown adipocytes. Journal of Biological Chemistry 285 7153-7164. (doi:10.1074/jbc.M109.053942)

Pisani DF, Djedaini M, Beranger GE, Elabd C, Scheideler M, Ailhaud G \& Amri EZ 2011 Differentiation of human adipose-derived stem cells into "Brite" (brown-in-white) adipocytes. Frontiers in Endocrinology 287. (doi:10.3389/fendo.2011.00087)

Rosenwald M, Perdikari A, Rulicke T \& Wolfrum C 2013 Bi-directional interconversion of brite and white adipocytes. Nature Cell Biology 15 659-667. (doi:10.1038/ncb2740)

Rothwell NJ \& Stock MJ 1983 Luxuskonsumption, diet-induced thermogenesis and brown fat: the case in favour. Clinical Science 64 19-23.

Sakurai H, Sakaguchi Y, Shoji E, Nishino T, Maki I, Sakai H, Hanaoka K, Kakizuka A \& Sehara-Fujisawa A 2012 In vitro modeling of paraxial mesodermal progenitors derived from induced pluripotent stem cells. PLOS ONE 7 e47078. (doi:10.1371/journal.pone.0047078)

Sanchez-Gurmaches J, Hung CM, Sparks CA, Tang Y, Li H \& Guertin DA 2012 PTEN loss in the Myf5 lineage redistributes body fat and reveals subsets of white adipocytes that arise from Myf5 precursors. Cell Metabolism 16 348-362. (doi:10.1016/j.cmet.2012.08.003)

Schulz TJ, Huang P, Huang TL, Xue R, McDougall LE, Townsend KL, Cypess AM, Mishina Y, Gussoni E \& Tseng YH 2013 Brown-fat paucity due to impaired BMP signalling induces compensatory browning of white fat. Nature 495 379-383. (doi:10.1038/nature11943)

Seale P, Bjork B, Yang W, Kajimura S, Chin S, Kuang S, Scime A, Devarakonda S, Conroe HM, Erdjument-Bromage H et al. 2008 PRDM16 controls a brown fat/skeletal muscle switch. Nature $\mathbf{4 5 4} 961-967$. (doi:10.1038/nature07182)

Shan T, Liang X, Bi P, Zhang P, Liu W \& Kuang S 2013 Distinct populations of adipogenic and myogenic Myf5-lineage progenitors in white adipose tissues. Journal of Lipid Research 54 2214-2224. (doi:10.1194/jlr. M038711)

Sharp LZ, Shinoda K, Ohno H, Scheel DW, Tomoda E, Ruiz L, Hu H, Wang L, Pavlova Z, Gilsanz V et al. 2012 Human BAT possesses molecular signatures that resemble beige/brite cells. PLOS ONE 7 e49452. (doi:10.1371/journal.pone.0049452)

Skurk T \& Hauner H 2012 Primary culture of human adipocyte precursor cells: expansion and differentiation. Methods in Molecular Biology $\mathbf{8 0 6}$ 215-226.

Tang W, Zeve D, Suh JM, Bosnakovski D, Kyba M, Hammer RE, Tallquist MD \& Graff JM 2008 White fat progenitor cells reside in the adipose vasculature. Science 322 583-586. (doi:10.1126/science.1156232)

Thorns C, Schardt C, Katenkamp D, Kahler C, Merz H \& Feller AC 2008 Hibernoma-like brown fat in the bone marrow: report of a unique case. Virchows Archiv 452 343-345. (doi:10.1007/s00428-007-0559-4)

Tran KV, Gealekman O, Frontini A, Zingaretti MC, Morroni M, Giordano A, Smorlesi A, Perugini J, De Matteis R, Sbarbati A et al. 2012 The vascular endothelium of the adipose tissue gives rise to both white and brown fat cells. Cell Metabolism 15 222-229. (doi:10.1016/j.cmet. 2012.01.008)

Tseng YH, Kokkotou E, Schulz TJ, Huang TL, Winnay JN, Taniguchi CM, Tran TT, Suzuki R, Espinoza DO, Yamamoto Y et al. 2008 New role of bone morphogenetic protein 7 in brown adipogenesis and energy expenditure. Nature 454 1000-1004. (doi:10.1038/ nature07221)

Walden TB, Hansen IR, Timmons JA, Cannon B \& Nedergaard J 2012 Recruited vs. nonrecruited molecular signatures of brown, "brite", and white adipose tissues. American Journal of Physiology. Endocrinology and Metabolism 302 E19-E31. (doi:10.1152/ajpendo.00249.2011)

Wu J, Bostrom P, Sparks LM, Ye L, Choi JH, Giang AH, Khandekar M, Virtanen KA, Nuutila P, Schaart G et al. 2012 Beige adipocytes are a distinct type of thermogenic fat cell in mouse and human. Cell $\mathbf{1 5 0}$ 366-376. (doi:10.1016/j.cell.2012.05.016)

Yin H, Pasut A, Soleimani VD, Bentzinger CF, Antoun G, Thorn S, Seale P, Fernando P, van Ijcken W, Grosveld F et al. 2013 MicroRNA-133 controls brown adipose determination in skeletal muscle satellite cells by targeting Prdm16. Cell Metabolism 17 210-224. (doi:10.1016/ j.cmet.2013.01.004)

Zilberfarb V, Pietri-Rouxel F, Jockers R, Krief S, Delouis C, Issad T \& Strosberg AD 1997 Human immortalized brown adipocytes express functional $\beta 3$-adrenoceptor coupled to lipolysis. Journal of Cell Science 110 801-807.

Zingaretti MC, Crosta F, Vitali A, Guerrieri M, Frontini A, Cannon B, Nedergaard J \& Cinti S 2009 The presence of UCP1 demonstrates that metabolically active adipose tissue in the neck of adult humans truly represents brown adipose tissue. FASEB Journal 23 3113-3120. (doi:10.1096/fj.09-133546)

Received in final form 3 September 2013

Accepted 16 September 2013

Accepted Preprint published online 16 September 2013 http://jme.endocrinology-journals.org DOI: 10.1530/JME-13-0158
() 2013 Society for Endocrinology Printed in Great Britain
Published by Bioscientifica Ltd 